\title{
On the Properties of Optimal Weak Links in Consensus Networks
}

\author{
Makan Fardad, Xi Zhang, Fu Lin, and Mihailo R. Jovanović
}

\begin{abstract}
We study consensus networks in which each node updates its state by taking a weighted average of the states of its neighbors. Our objective is to determine the optimal set of weak links whose addition to the network maximally improves the efficiency of reaching consensus. Allocating a small amount of resources to the entire network with which new links can be created, we employ a perturbation method to cast this problem as a linear program. We demonstrate that the set of optimal weak links is sparse and, based on extensive numerical experiments, conjecture that they are also long-range. Examples are provided to illustrate the utility of our developments.
\end{abstract}

Index Terms-Convex optimization, linear programming, long-range links, opinion dynamics, perturbation analysis, social networks, sparsity.

\section{INTRODUCTION}

Consensus is often of primary importance in collaborative networks: Generators in power networks synchronize and their synchrony can be thought of as consensus in the values of their frequency and phase [1]. Schools of fish achieve consensus in their directional heading [2]. Rendezvous of a robotic swarm can be considered as a consensus problem in both time and space [3]. Societies and communities strive to reach consensus on social issues. Other instances of consensus in networks include load balancing in parallel computing systems [4] and average consensus in sensor networks [5].

In this paper we use the DeGroot model [6], in which nodes update their values using a weighted average of their own current value and that of their neighbors. In this model, all nodes will eventually reach consensus if the network is connected. However, in the present work we are interested in the transient behavior of node values around a consensus state and the efficiency with which consensus is attained, rather than in the consensus value itself. We assume that there is an incentive in the network as a whole to create new links in order to facilitate the consensus process, and ask the following question: Given a small amount of resources with which to create new links, what are the best 'weak' links whose creation would maximize the efficiency of reaching consensus? Here, by weak links we imply those of small strength.

Financial support from the National Science Foundation under awards CNS-1329885 and ECCS-1407958 is gratefully acknowledged.

M. Fardad and X. Zhang are with the Department of Electrical Engineering and Computer Science, Syracuse University, Syracuse, NY 13244. F. Lin is with the Mathematics and Computer Science Division, Argonne National Laboratory, Argonne, IL 60439. M. R. Jovanović is with the Department of Electrical and Computer Engineering, University of Minnesota, Minneapolis, MN 55455. E-mails: makan@syr.edu, xzhang49@syr.edu, fulin@mcs.anl.gov, mihailo@umn.edu.
Perturbation methods constitute a powerful toolbox for uncovering important trends in the design of complex systems [7]-[9]. Recently, these methods have been effectively employed to enhance coherence in vehicular formations [10] and to control the onset of turbulence in shear flows [11], [12]. We employ perturbation techniques in the optimal weak link creation problem to recast it as a linear program. We demonstrate that the optimal weak links are both sparse and long-range. The cohesive power of weak ties and their pivotal role in the diffusion of information was illustrated in the influential work [13] in the context of social networks, where weak links can be interpreted as infrequent communication between nodes.

In comparison to existing literature, our work is most related to that of Boyd et al. [14] on fastest mixing Markov chains, Ghosh and Boyd on growing well-connected networks [15], Baras and Hovareshti [16] on efficient communication topologies in networked systems, and Olfati-Saber [17] on ultrafast consensus in small-world networks. We also draw on recent developments that have utilized $\ell_{1}$ regularization of optimal control problems as a means for designing sparse consensus [18], [19] and synchronization networks [20]. In all these works, attention is restricted to symmetric communication links corresponding to undirected graphs. In contrast, the communication structure of our network can be described by any strongly connected directed graph. Furthermore, in contrast to [14], we do not assume a predetermined communication architecture for the new links. In [16] the performance of the network is characterized by the number of spanning trees in the undirected graph that describes it. In this work, however, we measure performance using the cumulative behavior of node values around their consensus value. While in [17] new links result from a random process, here we obtain new links as the solution to an optimization problem. Finally, an analytical characterization of how the addition of cycles enhances the performance of undirected consensus networks has been provided in [19], whereas we provide a procedure for the identification of optimal weak links in directed consensus networks.

\section{Optimal Link CREATiOn in CONSENSUS NETWORKS}

In this section we describe the network dynamics, introduce a measure of collective performance, and formulate an optimization problem for the generation of new communication links. Our setup is based on that reported in [21]. Although the proposed framework has general applicability, we focus on social networks as a 
motivational application and concrete instance of consensus networks.

Consider a network of $n$ agents whose beliefs evolve according to the DeGroot model [6]

$$
x(k+1)=T x(k) .
$$

Here, $x(k)$ is a column $n$-vector composed of nonnegative values that represent the beliefs of the agents with regards to a particular social issue at time $k$, and $T$ is a (not necessarily symmetric) matrix that satisfies

$$
T \mathbb{1}=\mathbb{1}, \quad T \geq 0,
$$

where $\mathbb{1}$ is the column vector of all ones and the matrix inequality is elementwise. Assuming that all diagonal entries of $T$ are positive and that the graph described by $T$ is strongly connected, all eigenvalues of $T$ except for the one at $\lambda=1$ belong to the open unit disk. Furthermore, network (1)-(2) is guaranteed to reach consensus asymptotically [6], [22]. Finally, if the system is initialized at some $x(0)$ whose entries all belong to the interval $[0,1]$, then the entries of $x(k)$ remain within this interval for all time.

The paper [21] proposes a new measure of collective performance, in the context of the efficiency of reaching consensus, that quantifies collaborative behavior across time and space. This measure is independent of the actual consensus value and depends only on the cumulative transient behavior around it. To elaborate, let $Q$ be a symmetric matrix that satisfies

$$
\mathbb{1}^{T} Q \mathbb{1}=0, \quad \zeta^{T} Q \zeta>0 \text { for all } \zeta \neq 0 \text { s.t. } \zeta^{T} \mathbb{1}=0 .
$$

The properties of $Q$ ensure that $x(k)^{T} Q x(k)$ quantifies the deviation of the entries of $x(t)$ from a uniform value. Different $Q$ can be used to penalize different spatial scales of node-value variation across the network.

Let $e_{i}$ denote the $i$ th standard basis vector in $\mathbb{R}^{n}$, and let $x(0)=e_{i}$. This can be interpreted as a network in which all agents have zero initial value except for the $i$ th node, whose value is equal to one. From (1) it follows that the propagation of this initial value through the network is described by $x(k)=T^{k} e_{i}$, and $x(k)^{T} Q x(k)=e_{i}^{T} T^{k T} Q T^{k} e_{i}$ measures deviation-from-consensus at time $k$. Summing over $k, i$, and defining $J:=\sum_{i=1}^{n} \sum_{k=0}^{\infty} e_{i}^{T} T^{k T} Q T^{k} e_{i}$ as a cumulative measure of consensus-reaching efficiency, it can be shown that

$$
J=\operatorname{trace}(P),
$$

where $P \succeq 0$ satisfies the Lyapunov equation

$$
P=\left(T-\mathbb{1}^{T} / n\right)^{T} P\left(T-\mathbb{1}^{T} / n\right)+Q .
$$

The reason for subtracting $\mathbb{1} \mathbb{1}^{T} / n$ from $T$ is to eliminate the eigenvalue $\lambda=1$ of $T$ and render the spectrum of $T-1 \mathbb{1}^{T} / n$ inside the open unit disk. This guarantees that (4) always has a well-defined solution $P$. Since $T \mathbb{1}=\mathbb{1}$ and $Q \mathbb{1}=0$, the eigenvalue $\lambda=1$ of $T$ is unobservable through $Q$, and thus replacing $T$ by $T-1 \mathbb{1}^{T} / n$ does not affect the value of $J$. Finally, as alluded to earlier, the freedom in choosing $Q$ can be used to penalize different spatial scales of deviation-from-consensus, such as the sum of squares of node deviations from the global average or the sum of squares of node deviations from their local neighbors.

We now consider the optimal link creation problem [21],

$$
\begin{array}{ll}
\operatorname{minimize} & \operatorname{trace}(P) \\
\text { subject to } \quad P= & \left(S T+U-\mathbb{1}^{T} / n\right)^{T} P . \\
& \cdot\left(S T+U-\mathbb{1}^{T} / n\right)+Q, \quad P \mathbb{1}=0 \\
S= & I-\operatorname{diag}\{\sigma\}, \quad 0 \leq \sigma \leq \mathbb{1}, \quad \mathbb{1}^{T} \sigma \leq \varrho \\
U \mathbb{1}= & \sigma, \quad U \geq 0,
\end{array}
$$

where the optimization variables are the $n$-vector $\sigma$ and the $n \times n$ matrices $U, S, P$, and all matrix inequalities are elementwise. We next elaborate on the formulation of problem (5).

We assume that the network as a whole is allotted a total budget $\varrho$ with which to create new links, and that $\sigma_{i}$ denotes the sum of link weights corresponding to new links created by the $i$ th agent; thus, $\sum_{i=1}^{n} \sigma_{i} \leq \varrho$. Furthermore, for the $i$ th agent to create new links of total weight $\sigma_{i}$, it has to make space in its weighted averaging scheme by scaling down, by a factor of $1-\sigma_{i}$, the weight it places on its existing links. The elementwise-nonnegative matrix $U$ describes the weight and distribution of the new links; the $(i, j)$ th entry of $U$ signifies a new link made by agent $i$ to agent $j$. Consequently, the entries in the $i$ th row of $U$ sum to $\sigma_{i}$. Opinions in this augmented network evolve according to (1) in which $T$ is replaced by $S T+U$ and $S=\operatorname{diag}\left\{1-\sigma_{i}, i=1, \ldots, n\right\}$. The efficiency of this augmented network in achieving consensus is captured by $\operatorname{trace}(P)$, where $P$ satisfies (4) with $T$ is replaced by $S T+U$.

We bring attention to the fact that the number of links is not explicitly penalized in (5). Therefore, the possibilities in the solution of (5) range from all agents making many new links with small weights (corresponding to a full $U$ with many small entries) to some agents making a few links with large weights (corresponding to a sparse $U$ with a few large entries). In the sections that follow, we will demonstrate that solutions are indeed sparse.

Defining the diagonal matrix $D$

$$
D=\operatorname{diag}\{\sigma\}
$$

which in particular implies $D \mathbb{1}=\sigma$, problem (5) can be equivalently written as

$$
\begin{array}{ll}
\operatorname{minimize} & \operatorname{trace}(P) \\
\text { subject to } \quad P= & \left(S T+U-\mathbb{1}^{T} / n\right)^{T} P . \\
& \cdot\left(S T+U-\mathbb{1}^{T} / n\right)+Q, \quad P \mathbb{1}=0 \\
S= & I-D, \quad 0 \leq D \leq I, \quad \operatorname{trace}(D) \leq \varrho \\
U \mathbb{1}= & D \mathbb{1}, \quad U \geq 0,
\end{array}
$$


where the optimization variables are the matrices $U, D, S, P$.

\section{SPARSITY OF OPTIMAL WEAK LINKS}

In this section we consider the problem of finding optimal weak links. We refer to a link as weak if it has small weight, and consider such links as modeling infrequent communication between nodes. We demonstrate that such links have the desirable property of being sparse.

In what follows, we approach problem (6) from a perturbation standpoint. We assume that the sum of weights $\varrho$ and the entries of the diagonal matrix $D$ are all small positive numbers. This also implies that the elementwisenonnegative matrix $U$ has small entries. We thus consider the proceeding problem in which we seek the optimal weak links to be created

$$
\begin{array}{ll}
\operatorname{minimize} & \operatorname{trace}(P) \\
\text { subject to } \quad P= & \left(S T+U-\mathbb{1} \mathbb{1}^{T} / n\right)^{T} P . \\
& \cdot\left(S T+U-\mathbb{1}^{T} / n\right)+Q, \quad P \mathbb{1}=0 \\
S= & I-D, \quad D \text { diagonal, } \operatorname{trace}(D) \leq \varepsilon \\
& U \mathbb{1}=D \mathbb{1}, \quad U \geq 0,
\end{array}
$$

where $\varepsilon$ is a small positive number. ${ }^{1}$ The constraint $\operatorname{trace}(D) \leq \varepsilon$ implies that, given a total budget of $\varepsilon$, the scenarios for making new links range from one node making one link with weight $\varepsilon$ to many nodes making many links with weights that add up to $\varepsilon$.

Proposition 1: For small enough values of $\varepsilon>0$, the solution of (7) can be determined from the solution of

$$
\begin{array}{ll}
\text { minimize } & \operatorname{trace}\left(K^{T}(U-D T)\right) \\
\text { subject to } & U \mathbb{1}=D \mathbb{1}, \quad U \geq 0 \\
& \operatorname{trace}(D) \leq 1, \quad D \text { diagonal, }
\end{array}
$$

where the optimization variables are the matrices $U$ and $D$, and $K$ is a fixed matrix that depends on $T$ and $Q$.

Before we give the proof of the proposition, we elaborate on how to use the solution of (8) to obtain the solution of (7). Let $\varrho$ be a given small number, signifying the total weight of the new links to be created. Set $\varepsilon=\varrho$, and solve (8) to obtain the optimal $U^{*}, D^{*}$. Then, with an abuse of notation, the matrices $U:=\varepsilon U^{*}$ and $D:=\varepsilon D^{*}$ solve (7) for small enough values of $\varepsilon$.

Proof: With an abuse of notation, we replace $D, S, U$, and $P$ in (7) with $\varepsilon D, I-\varepsilon D, \varepsilon U$, and

$$
P=P_{0}+\varepsilon P_{1}+\varepsilon^{2} P_{2}+\cdots,
$$

respectively. Substituting in the Lyapunov equation and collecting terms gives

$$
P_{i}=\left(T-\mathbb{1}^{T} / n\right)^{T} P_{i}\left(T-\mathbb{1}^{T} / n\right)+Q_{i}, \quad i=0,1,
$$

\footnotetext{
${ }^{1}$ The constraint $0 \leq D \leq I$ has been omitted from (7), since $D \geq 0$ is guaranteed by $\{U \mathbb{1}=D \mathbb{1}, U \geq 0, D$ diagonal $\}$, and $D \leq \bar{I}$ is guaranteed by $\{\operatorname{trace}(D) \leq \varepsilon, D$ diagonal, $\varepsilon \ll 1\}$.
}

with

$$
\begin{aligned}
Q_{0}:= & Q, \\
Q_{1}:= & (-D T+U)^{T} P_{0}\left(T-\mathbb{1}^{T} / n\right) \\
& +\left(T-\mathbb{1}^{T} / n\right)^{T} P_{0}(-D T+U) .
\end{aligned}
$$

We point out that $Q_{1}$ depends on $P_{0}$. On the other hand, the objective function of our optimization problem can now be rewritten as

$$
\operatorname{trace}(P)=\operatorname{trace}\left(P_{0}\right)+\varepsilon \operatorname{trace}\left(P_{1}\right)+O\left(\varepsilon^{2}\right) .
$$

Since $P_{0}$ has no dependence on the optimization variables, its trace has a constant value and can be eliminated from the objective. Also, given that $\varepsilon$ takes only small values, we will disregard the higher-order terms and hereafter consider $\operatorname{trace}\left(P_{1}\right)$ as our new minimization objective.

The spectrum of $T-\mathbb{1}^{T} / n$ is guaranteed to belong to the open unit disk and hence the Lyapunov equations for $P_{0}$ and $P_{1}$ admit the unique solutions

$$
P_{i}=\sum_{k=0}^{\infty}\left(T-\mathbb{1}^{T} / n\right)^{k T} Q_{i}\left(T-\mathbb{1}^{T} / n\right)^{k}, \quad i=0,1 .
$$

It is easy to show that $P_{0} \mathbb{1}=P_{1} \mathbb{1}=0$. Using the properties of the trace, our new objective function can be rewritten as

$$
\begin{aligned}
\operatorname{trace}\left(P_{1}\right) & =\sum_{k=0}^{\infty} \operatorname{trace}\left(\left(T-\mathbb{1}^{T} / n\right)^{k}\left(T-\mathbb{1}^{T} / n\right)^{k T} Q_{1}\right) \\
& =\operatorname{trace}\left(H Q_{1}\right),
\end{aligned}
$$

where

$$
H:=\sum_{k=0}^{\infty}\left(T-\mathbb{1}^{T} / n\right)^{k}\left(T-\mathbb{1} \mathbb{1}^{T} / n\right)^{k T} .
$$

The matrix $H$ can also be found as the solution to the Lyapunov equation

$$
H=\left(T-\mathbb{1}^{T} / n\right) H\left(T-\mathbb{1} \mathbb{1}^{T} / n\right)^{T}+I
$$

Further simplification of $\operatorname{trace}\left(P_{1}\right)$ using the definition of $Q_{1}$ yields

$$
\begin{aligned}
\operatorname{trace}\left(P_{1}\right) & =2 \operatorname{trace}\left(H\left(T-\mathbb{1}^{T} / n\right)^{T} P_{0}(U-D T)\right) \\
& =2 \operatorname{trace}\left(K^{T}(U-D T)\right),
\end{aligned}
$$

where

$$
K:=P_{0}\left(T-\mathbb{1}^{T} / n\right) H .
$$

The problem for the identification of optimal weak links can now be stated as

$$
\begin{array}{ll}
\operatorname{minimize} & \operatorname{trace}\left(K^{T}(U-D T)\right) \\
\text { subject to } & U \mathbb{1}=D \mathbb{1}, \quad U \geq 0 \\
& \operatorname{trace}(D) \leq 1, \quad D \text { diagonal, }
\end{array}
$$

where the optimization variables are the matrices $U$ and $D$. This problem has a linear objective and linear constraints and is thus a linear program. The proof of the proposition is now complete. 
The following proposition is one of the main results of this work. Let $\operatorname{card}(X)$ and $\|X\|_{\ell_{1}}$ respectively denote the cardinality and the 1 -norm of the matrix/vector $X$,

$$
\begin{aligned}
\operatorname{card}(X) & : \text { number of nonzero entries in } X, \\
\|X\|_{\ell_{1}} & :=\sum_{i, j}\left|X_{i j}\right| .
\end{aligned}
$$

Proposition 2: Problem (8) is equivalent to

$$
\begin{aligned}
& \text { minimize } \operatorname{trace}\left((K-F)^{T} U\right) \\
& \text { subject to }\|U\|_{\ell_{1}} \leq 1, \quad U \geq 0,
\end{aligned}
$$

where the optimization variable is the matrix $U$, and $F$ is a fixed matrix that depends on $T$ and $K$. Moreover, problem (9) has a sparse solution $U^{*}$ that satisfies $\operatorname{card}\left(U^{*}\right) \leq 1$.

Proof: Using the diagonality of $D$ and the constraint $U \mathbb{1}=D \mathbb{1}$, the objective of (8) can be rewritten as

$$
\begin{aligned}
\operatorname{trace}\left(K^{T}(U-D T)\right) & =\operatorname{trace}\left(U K^{T}-D T K^{T}\right) \\
& =\operatorname{trace}\left(U K^{T}-U F^{T}\right)
\end{aligned}
$$

where

$$
F \text { : matrix whose } i \text { th row is equal to }\left(K T^{T}\right)_{i i} \mathbb{1}^{T},
$$

and $\left(K T^{T}\right)_{i i}$ is the $i$ th diagonal entry of $K T^{T}$. On the other hand, the remaining constraints in (8) can be rewritten as

$$
\|U\|_{\ell_{1}} \leq 1, \quad U \geq 0 .
$$

Problem (8) thus becomes

$$
\begin{array}{ll}
\operatorname{minimize} & \operatorname{trace}\left((K-F)^{T} U\right) \\
\text { subject to } & \|U\|_{\ell_{1}} \leq 1, \quad U \geq 0 .
\end{array}
$$

Let $\nu$ denote the value of the smallest entry of the matrix $K-F$,

$$
\nu=\min \left\{K_{i j}-F_{i j}, i, j=1, \ldots, n\right\},
$$

and let $\left(i_{\nu}, j_{\nu}\right)$ denote the indices of any entry of $K-F$ that achieves this minimum. If $\nu \geq 0$, then clearly $U^{*}=0$, as any other admissible $U$ will render a positive value of the objective. If $\nu<0$, then let $U^{*}$ be defined by its entries as

$$
U_{i j}^{*}= \begin{cases}0 & (i, j) \neq\left(i_{\nu}, j_{\nu}\right) \\ 1 & (i, j)=\left(i_{\nu}, j_{\nu}\right)\end{cases}
$$

for $i, j=1, \ldots, n$. This $U^{*}$ achieves the smallest value of the objective among all nonzero and elementwise-nonnegative $U$ whose 1-norm is bounded by one. The proof of the proposition is now complete.

We note that Proposition 2 does not guarantee a unique solution; indeed, the solution is only unique if the minimum $\nu$ in the proof of the proposition is achieved by only one entry of $K-F$. For example, if the network is spatially invariant, then $K$ and $F$ are circulant and there are at least $n$ entries of the matrix $K-F$ that achieve the minimum $\nu$. This leads to an entire family of sparse solutions. We will elaborate on such solutions in the next section.

\section{Optimal Weak Links IN SPATIALLY INVARIANT NETWORKS}

In this section we assume that the network, and the measure used to quantify its consensus efficiency, are spatially invariant. We demonstrate that the optimal weak links inherit the spatial invariance property while remaining sparse. Furthermore, based on extensive numerical experiments, we conjecture that these weak links are also long-range.

In what follows, we assume a spatially invariant network described by a circulant matrix $T$. We also assume that $Q$ is circulant. To justify these choices for $T$ and $Q$ we note that our developments in this section are motivated by the work of Watts and Strogatz [23] on small-world networks, where the authors consider random 'perturbations' (in the form of rewiring of links) to regular connected graphs composed of local links. We take such regular graphs to be represented by circulant matrices $T$. Furthermore, as far as (democratic) consensus is concerned, most relevant performance measures use $Q$ matrices that are inherently spatially invariant (as there is no reason to prefer certain nodes over others), e.g.,

$x^{T} Q_{\text {circ }} x=\left(x_{1}-x_{2}\right)^{2}+\cdots+\left(x_{n-1}-x_{n}\right)^{2}+\left(x_{n}-x_{1}\right)^{2}$, $x^{T} Q_{\text {ave }} x=\left(x_{1}-x_{\text {ave }}\right)^{2}+\cdots+\left(x_{n}-x_{\text {ave }}\right)^{2}$,

with $x_{\text {ave }}:=(1 / n) \sum_{i} x_{i}$. Indeed, the latter quadratic form is commonly used and corresponds to $Q=I-\mathbb{1}^{T} / n$.

The circulant assumption on $T$ and $Q$ allows us to find closed-form expressions for $P_{0}, K$, and $H$,

$$
\begin{aligned}
P_{0} & =H Q, \quad K=H^{2} Q\left(T-\mathbb{1}^{T} / n\right), \\
H & =\left(I-\left(T-\mathbb{1}^{T} / n\right)\left(T-\mathbb{1}^{T} / n\right)^{T}\right)^{-1} .
\end{aligned}
$$

To obtain these expressions, we have used the fact that all circulant matrices of the same dimension commute. Since the set of circulant matrices of dimension $n$ is closed under the operations of summation, multiplication, and inversion, the matrices $P_{0}, K$, and $H$ are also circulant.

Proposition 3: Under the assumption of circulant $T$ and $Q$, there exists a solution $U^{*}, D^{*}$ to problem (8) which has the property that $U^{*}$ is circulant and $D^{*}$ is a scalar multiple of the identity.

Proof: The proof follows from the observation that both the objective and the constraints in (8) are shift invariant. We omit the details due to space limitations.

Proposition 3 states that (8) always has a circulant solution $U$. Thus, without sacrificing performance we can reduce the dimension of the search space by restricting $U$ to the class of circulant matrices. Under the assumption of circulant $U$, problem (8) simplifies to

$$
\begin{array}{ll}
\operatorname{minimize} & \operatorname{trace}\left(K^{T}(U-d T)\right) \\
\text { subject to } & U \mathbb{1}=d \mathbb{1}, \quad U \geq 0 \\
& d \leq 1 / n, \quad U \text { circulant, }
\end{array}
$$

where the optimization variables are the matrix $U$ and the 
scalar $d$. In this setting, all nodes are allocated the same amount of weight with which to make new links, and each can choose to either make many small links or just a few large ones.

Next, we exploit the circulant structure of all matrices involved in the above problem to further simplify its formulation. Let $\mu, \tau, \kappa$, respectively denote the first columns of $U, T, K$. Then the objective function in the preceding program becomes

$$
\operatorname{trace}\left(K^{T} U-d K^{T} T\right)=n \kappa^{T}(\mu-d \tau),
$$

and the constraints can be rewritten as

$$
\mathbb{1}^{T} \mu=d, \quad \mu \geq 0, \quad d \leq 1 / n .
$$

We thus have

$$
\begin{array}{ll}
\operatorname{minimize} & \kappa^{T}(\mu-d \tau) \\
\text { subject to } & \mathbb{1}^{T} \mu=d, \quad \mu \geq 0, \quad d \leq 1 / n,
\end{array}
$$

or equivalently

$$
\begin{array}{ll}
\operatorname{minimize} & (\kappa-\varphi)^{T} \mu \\
\text { subject to } & \|\mu\|_{\ell_{1}} \leq 1 / n, \quad \mu \geq 0,
\end{array}
$$

where

$$
\varphi:=\left(\tau^{T} \kappa\right) \mathbb{1}
$$

Problem (10) is a linear program and can be solved efficiently for very large systems. Not surprisingly, the solution $\mu^{*}$ is sparse.

Proposition 4: Problem (10) has a sparse solution $\mu^{*}$ that satisfies $\operatorname{card}\left(\mu^{*}\right) \leq 1$.

Proof: The proof is similar to that of Prop. 2.

Our extensive numerical experiments evidence that the solution of (10) always corresponds to the generation of long-range links; see Section V for examples. We thus make the following conjecture.

Conjecture 5: Under the assumption of circulant $T$ and $Q$, any solution $U^{*}$ of optimization problem (8) corresponds to the generation of long-range links.

Indeed, we can prove the conjecture for the special case of circulant symmetric $T$ and $Q$ and in the limit of large $n$.

\section{Illustrative EXAMPLES}

We illustrate the sparse and long-range properties of the optimal weak links through some illustrative examples. For all computations we used CVX, a package for specifying and solving convex programs [24], [25].

Example 1: Consider a graph with $n=10$ and nearest neighbor interactions described by the circulant matrix $T$ with first column $\tau$,

$$
\tau=\left[\begin{array}{llllllllll}
\frac{10}{20} & \frac{3}{20} & 0 & 0 & 0 & 0 & 0 & 0 & 0 & \frac{7}{20}
\end{array}\right]^{T} .
$$

Let $Q=I-\mathbb{1}^{T} / n$. It can be seen from Fig. 1 that the optimal $\mu$ is given by

$$
\mu^{*}=\left[\begin{array}{llllllllll}
0 & 0 & 0 & 0 & 0 & \frac{1}{n} & 0 & 0 & 0 & 0
\end{array}\right]^{T},
$$

where the element 1 appears in the 6th entry, and corresponds to sparse long-range links. Indeed, each node makes a link to the node farthest away from itself; see Fig. 2. These links maximally reduce the graph diameter.

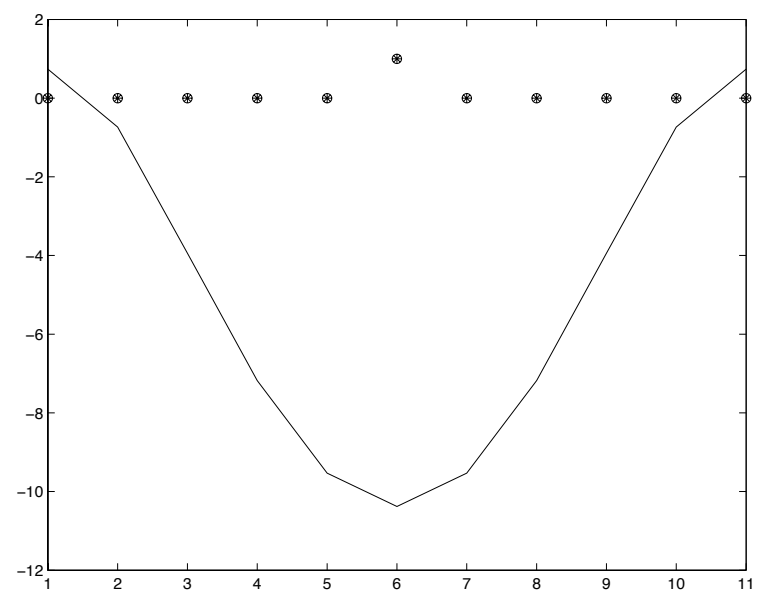

Fig. 1: The horizontal axis represents the indices of the entries of a vector in $\mathbb{R}^{n}$; the first entry repeats at the end to make the plot symmetric. The continuous plot gives the values of the entries of $\kappa-\varphi$; the circular points give the values of the entries of $n \mu^{*}$.

Example 2: Consider a graph with $n=10$ and with existing long-range links, described by the circulant matrix $T$ with first column $\tau$,

$$
\tau=\left[\begin{array}{llllllllll}
\frac{10}{20} & 0 & 0 & 0 & 0 & \frac{3}{20} & 0 & 0 & 0 & \frac{7}{20}
\end{array}\right]^{T} .
$$

Let $Q=I-\mathbb{1}^{T} / n$. Fig. 3 demonstrates that the optimal $\mu$ is given by

$$
\mu^{*}=\left[\begin{array}{llllllllll}
0 & 0 & \frac{1}{2 n} & 0 & 0 & 0 & 0 & \frac{1}{2 n} & 0 & 0
\end{array}\right]^{T},
$$

rendering sparse long-range links. It is interesting that the new links fall exactly in between existing links; see Fig. 4.

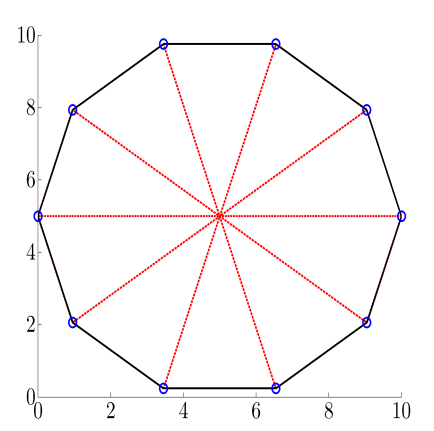

Fig. 2: Creation of sparse long-range links (red dashed lines) corresponds to solution $\mu^{*}$ in Fig. 1. Each node creates a link to connect to the farthest node. 
Thus, as in the previous example, the new links are created such that the diameter of the graph is maximally reduced.

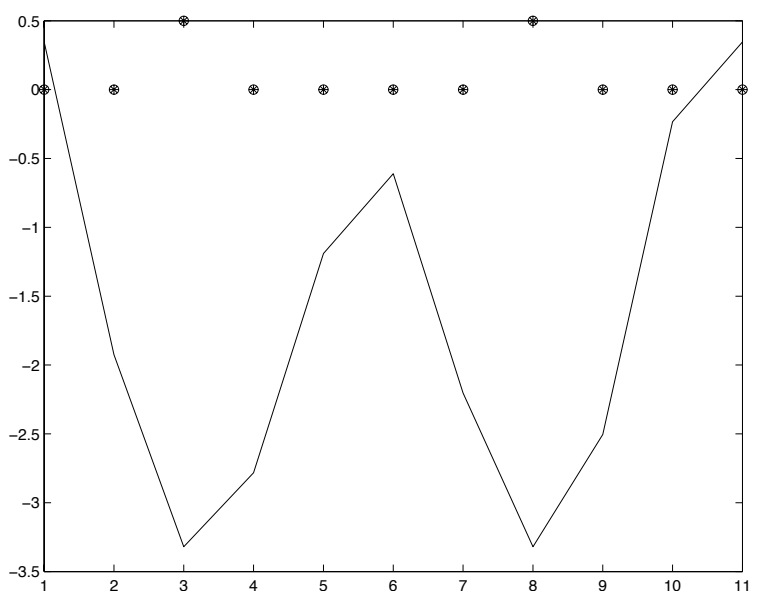

Fig. 3: The horizontal axis represents the indices of the entries of a vector in $\mathbb{R}^{n}$; the first entry repeats at the end to make the plot symmetric. The continuous plot gives the values of the entries of $\kappa-\varphi$; the circular points give the values of the entries of $n \mu^{*}$.

\section{CONCLUding REMARKS}

We study the creation of weak links that maximally improve the efficiency of reaching consensus in the DeGroot model. When the amount of resources available for the creation of new links is small, we employ first-order perturbation methods to show that the optimal weak links are sparse. Furthermore, for spatially invariant networks, we observe that the sparse weak links are also long-range. We demonstrate the utility of our results via illustrative examples. Our future work involves providing a proof for the long-range property of optimal weak links for general classes of spatially invariant networks.

\section{REFERENCES}

[1] F. Dorfler and F. Bullo, "Synchronization and transient stability in power networks and non-uniform Kuramoto oscillators," SIAM Journal on Control and Optimization, vol. 50, pp. 1616-1642, 2012.

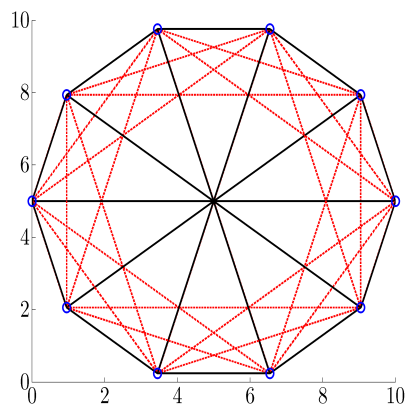

Fig. 4: Creation of sparse long-range links (red dashed lines) corresponds to solution $\mu^{*}$ in Fig. 3. Each node creates two links that fall in between existing links (black solid lines).
[2] A. Jadbabaie, J. Lin, and A. Morse, "Coordination of groups of mobile autonomous agents using nearest neighbor rules," IEEE Transactions on Automatic Control, vol. 48, no. 6, pp. 988-1001, June 2003.

[3] F. Bullo, J. Cortés, and S. Martínez, Distributed Control of Robotic Networks. Princeton University Press, 2009.

[4] L. Xiao, S. Boyd, and S.-J. Kim, "Distributed average consensus with least-mean-square deviation," Journal of Parallel and Distributed Computing, vol. 67, no. 1, pp. 33-46, 2007.

[5] S. Boyd, A. Ghosh, B. Prabhakar, and D. Shah, "Randomized gossip algorithms," IEEE Transactions on Information Theory, vol. 52, pp. 2508-2530, 2006.

[6] M. H. DeGroot, "Reaching a consensus," Journal of the American Statistical Association, vol. 69, pp. 118-121, 1974.

[7] M. Fardad and B. Bamieh, "Perturbation methods in stability and norm analysis of spatially periodic systems," SIAM Journal on Control and Optimization, vol. 47, no. 2, pp. 997-1021, 2008

[8] M. R. Jovanović and M. Fardad, " $H^{2}$ norm of linear time-periodic systems: A perturbation analysis," Automatica, vol. 44, pp. 2090-2098, 2008

[9] M. Fardad, F. Lin, and M. R. Jovanović, "On the optimal design of structured feedback gains for interconnected systems," in Proceedings of the 48th IEEE Conference on Decision and Control, 2009, pp. 978983.

[10] F. Lin, M. Fardad, and M. R. Jovanović, "Optimal control of vehicular formations with nearest neighbor interactions," IEEE Trans. Automat. Control, vol. 57, no. 9, pp. 2203-2218, 2012.

[11] R. Moarref and M. R. Jovanović, "Controlling the onset of turbulence by streamwise traveling waves. Part 1: Receptivity analysis," J. Fluid Mech., vol. 663, pp. 70-99, 2010.

[12] B. K. Lieu, R. Moarref, and M. R. Jovanović, "Controlling the onset of turbulence by streamwise traveling waves. Part 2: Direct numerical simulations," J. Fluid Mech., vol. 663, pp. 100-119, 2010.

[13] M. Granovetter, "The strength of weak ties," American Journal of Sociology, vol. 78, pp. 1360-1380, 1973.

[14] S. Boyd, P. Diaconis, P. Parrilo, and L. Xiao, "Fastest mixing markov chain on graphs with symmetries," SIAM Journal on Optimization, vol. 20, pp. 792-819, 2009.

[15] A. Ghosh and S. Boyd, "Growing well-connected graphs," in Proceedings of the 45th IEEE Conference on Decision and Control, 2006, pp. 6605-6611.

[16] J. S. Baras and P. Hovareshti, "Efficient and robust communication topologies for distributed decision making in networked systems," in Proceedings of the 48th IEEE Conference on Decision and Control, 2009, pp. 3751-3756.

[17] R. Olfati-Saber, "Ultrafast consensus in small-world networks," in Proceedings of the 2005 American Control Conference, 2005, pp. 2371-2378

[18] F. Lin, M. Fardad, and M. R. Jovanović, "Identification of sparse communication graphs in consensus networks," in Proceedings of the 50th Annual Allerton Conference on Communication, Control, and Computing, Monticello, IL, 2012, pp. 85-89.

[19] D. Zelazo, S. Schuler, and F. Allgöwer, "Performance and design of cycles in consensus networks," Systems and Control Letters, vol. 62, no. 1 , pp. 85-96, 2013

[20] M. Fardad, F. Lin, and M. R. Jovanović, "Design of optimal sparse interconnection graphs for synchronization of oscillator networks," IEEE Trans. Automat. Control, vol. 59, no. 9, pp. 2457-2462, 2014.

[21] M. Fardad, F. Lin, and M. R. Jovanović, "On optimal link creation for facilitation of consensus in social networks," in Proceedings of the 2014 American Control Conference, 2014, pp. 3790-3795.

[22] F. Fagnani and S. Zampieri, "Randomized consensus algorithms over large scale networks," IEEE Journal on Selected Areas in Communications, vol. 26, pp. 634-649, 2008.

[23] D. J. Watts and S. H. Strogatz, "Collective dynamics of 'small-world' networks," Nature, vol. 393, pp. 440-442, 1998.

[24] M. Grant and S. Boyd, "CVX: Matlab software for disciplined convex programming, version 2.0 beta," http://cvxr.com/cvx/, 2013.

[25] M. Grant and S. Boyd, "Graph implementations for nonsmooth convex programs," in Recent Advances in Learning and Control, ser. Lecture Notes in Control and Information Sciences, V. Blondel, S. Boyd, and H. Kimura, Eds. Springer-Verlag Limited, 2008, pp. 95-110. 